\title{
Study on the Microstructure Properties of Titanium Metal Crown Base
}

\section{Laser Rapid Forming}

\author{
Yulei Li ${ }^{\mathrm{a}}$, Jinjun Tang ${ }^{\mathrm{b}}$,Yuelai Dai ${ }^{\mathrm{c}}$, Xiaoyu Jin ${ }^{\mathrm{d}}$, Qun Wang ${ }^{\mathrm{e}}$
}

Ningbo Sub-academy of the National Weapons Science Research Academy, Ningbo 315103,China

aliyulei7395@163.com, bjinjun.tang@163.com, daiyuelai_1988@126.com,

dinxiaoyu_nb@163.com, ewangqun_yz@163.com

Key words: laser rapid forming; titanium; metallurgical; organizational performance

Abstract: This paper researches the influence of laser rapid prototyping process for titanium metal crown. molding quality using the microstructure. The results show that laser rapid prototyping technology can form a uniform, defect-free titanium base crown. And than the thickness of choice is crucial, relatively speaking, other process parameters on material structure is weak.

\section{Introduction}

Laser rapid forming was advanced manufacturing technology from newly developed in recent years. This technique can directly produce full density, high-precision[1,2], functional metal parts through a high-power laser sintering of molten metal powders. Currently, it has been a hot research for preparation techniques at home and abroad. Titanium and its alloys processing methods currently limited to casting, CNC machining and other types of processing methods, various methods have certain disadvantages and limitations[3,4]. There are some problems limit the application of titanium and titanium alloys, such as the casting has complicated process, casting defects and other issues, CNC machining method is not only a waste of material and can not complete the form more complex objects. Therefore, the laser rapid forming as a new way of processing, is to improve the existing methods and new ways to promote the development of titanium and its alloys[5].

This will be Titanium metal crown for the study, by optical experiments, observe the microstructure and properties of laser rapid forming titanium metal. And than from the microscopic point of view, analyze the impact of the Laser Rapid Forming Process to Titanium sample quality.

\section{Materials and Methods}

Laser rapid forming technology can form different ingredients and organizations at different sites of parts to has a reasonable control. As the laser rapid forming manufacturing almost point by point, so you can laser deposition in component parts of any part needed to achieve the best mix of parts with materials and performance. Laser rapid forming not only concerned about shaping precision and manufacturing speed of parts, while more attention to obtain excellent performance.

Rapid melting and solidification process from high-energy laser make the material dense and fine, thereby improving the overall performance of the material. The digital model was in Figure 1 . The base crown complex shapes, made of laser rapid forming, model design based on the process parameters in Table 1.

Experiment in coping cut from the middle, to observe the quality of the base crown molding, as shown in FIG. After inlay sample, according to the metallurgical requirements of the interface is 
polished, complete the production of metallurgical samples. The metallurgical samples after corrosion, observed metal structure in optical microscope.

Table 1 Process parameter control factors

\begin{tabular}{|c|c|c|c|}
\hline & Laser Power(w) & Scan $\operatorname{speed}(\mathrm{mm} / \mathrm{s})$ & Thickness $(\mathrm{mm})$ \\
\hline 1 & 150 & 2000 & 0.1 \\
\hline 2 & 100 & 1500 & 0.2 \\
\hline
\end{tabular}
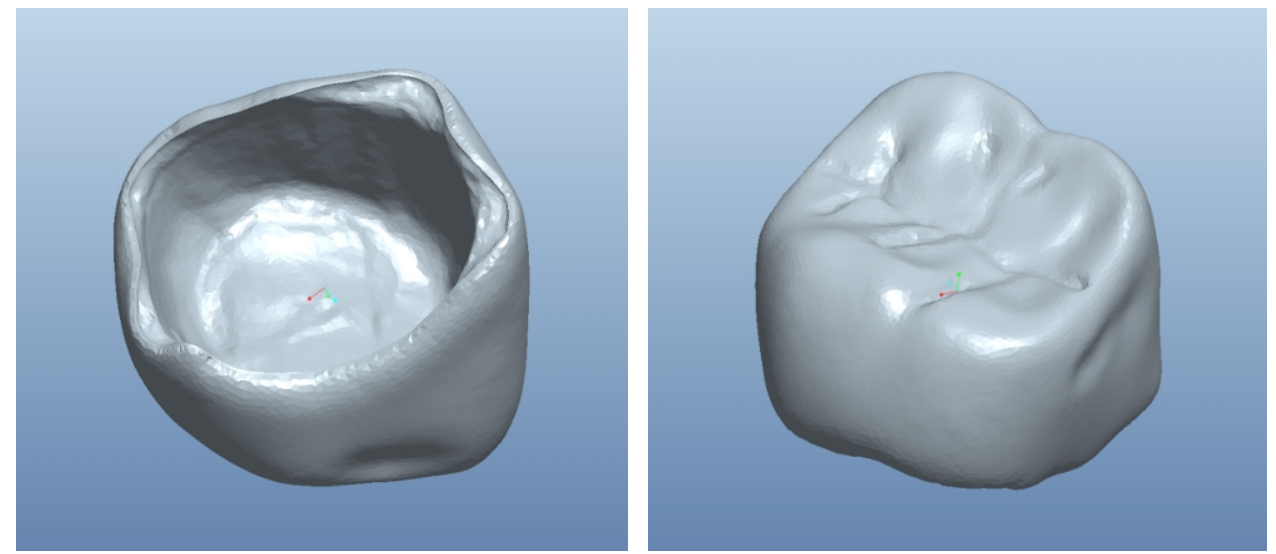

Fig. 1 The digital model

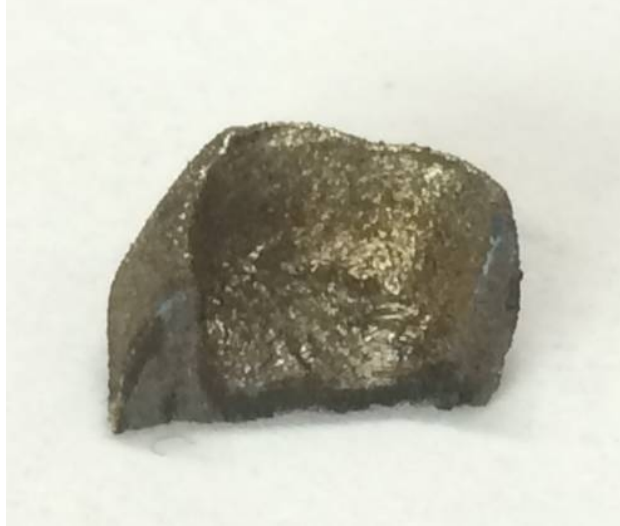

Fig. 2 Coping sectional

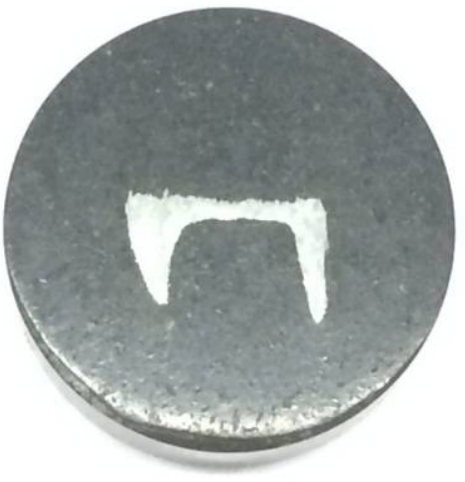

Fig. 3 Coping metallurgical samples

\section{Results and discussion}

Figure 4 shows the metallurgical microstructure of titanium crown with laser rapid forming, microstructure uniformity, no pores, cracks and other defects. $\alpha$ and $\beta$ phase interwoven into a basket-like organization for the rapid solidification conditions under typical morphology of titanium.

Laser power, scanning speed, thickness was important parameter in the process of rapid formation. The higher the laser power, the greater laser energy of per unit area, the larger area of the formed molten pool, so had the larger impact on the surface of fine size requirements. Copings complex shape, can't to use too much power. With the improvement of scanning speed, the amount of powder into the bath unit time will be reduced. And also improve the scanning speed will be reduced the interaction time of per unit area substrate and laser, thus affecting the quality of the 
specimen forming. The thickness selecting was relation to the forming precision Z-axis direction. Multilayer cladding, each layer cladding substrate preheat temperature cumulative effect situation is different, resulting in different forming quality. According to the results of Figure 4, the process parameters on the quality of the laser rapid forming have a certain influence, but within a certain range of small amplitude fluctuations, the impact is not great.

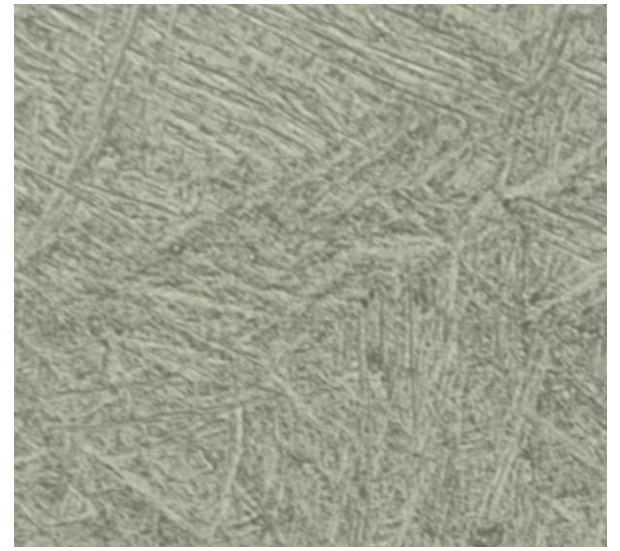

(a) $150 \mathrm{w} ; 2000 \mathrm{~mm} / \mathrm{s} ; 0.1 \mathrm{~mm}$

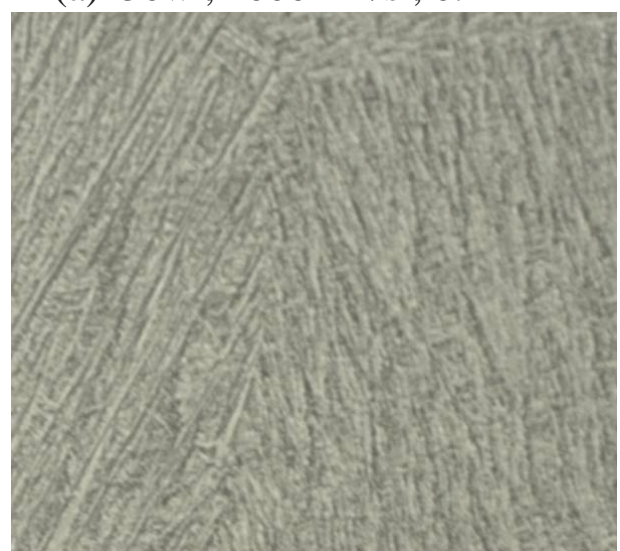

(c) $150 \mathrm{w} ; 1500 \mathrm{~mm} / \mathrm{s} ; 0.2 \mathrm{~mm}$

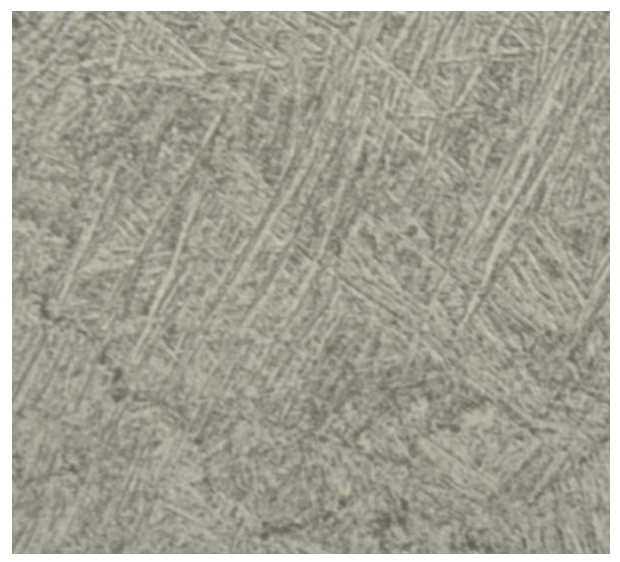

(e) $100 \mathrm{w} ; 2000 \mathrm{~mm} / \mathrm{s} ; 0.1 \mathrm{~mm}$

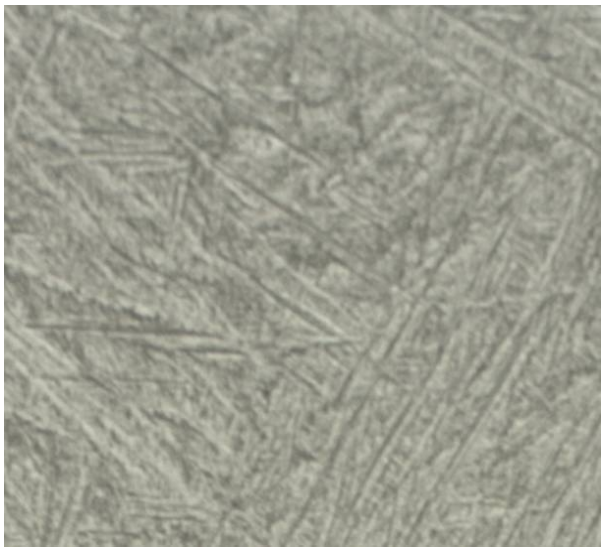

(b) $150 \mathrm{w} ; 2000 \mathrm{~mm} / \mathrm{s} ; 0.2 \mathrm{~mm}$

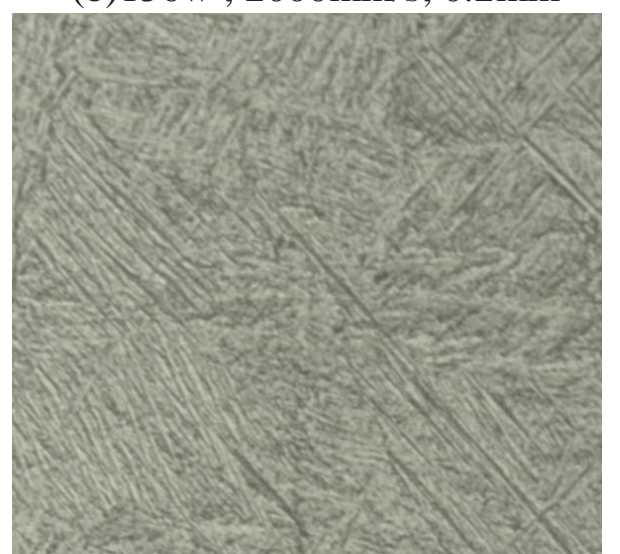

(d) $100 \mathrm{w} ; 1500 \mathrm{~mm} / \mathrm{s} ; 0.2 \mathrm{~mm}$

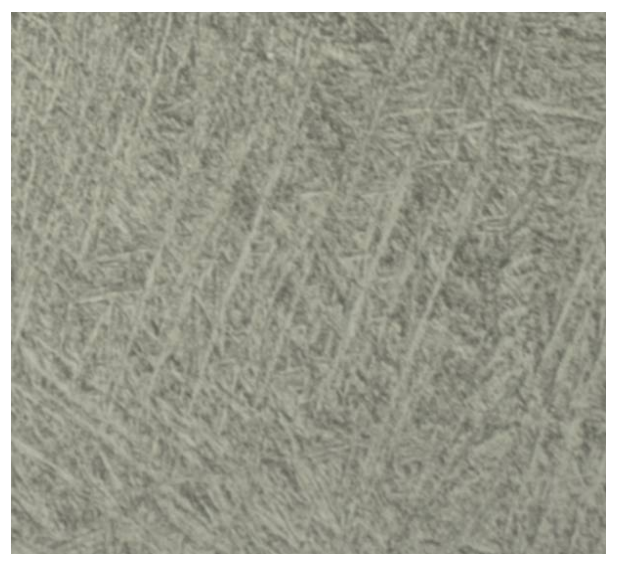

(f) $100 \mathrm{w} ; 1500 \mathrm{~mm} / \mathrm{s} ; 0.1 \mathrm{~mm}$

Fig. 4 Microstructure under different circumstances-500X

Laser rapid forming technology is laminated Manufacturing Technology. Relatively speaking, the choice of thickness is the most important parameters. When the thickness is too large, there is a 
certain amount of deviation between the deposited layer. While continuing cladding deviation further increases, eventually leading to the metal powder is not sufficiently melted to cause interlayer defects. It was right in theory the smaller the thickness, the higher the precision. However, in fact there was some problem with too thin thickness, on the one hand will result in lower processing efficiency, on the other hand due to the presence of the laser so that the actual thickness of the pool easily exceed the expected thickness, and ultimately affect the accuracy of processing.

\section{Conclusion}

1.Laser Rapid Forming titanium microstructure of $\alpha$ and $\beta$ phase interwoven into a basket-like tissue, is typical of rapid solidification of titanium tissue morphology affected laser power, scanning speed, thickness and so on.

2. Laser Rapid Forming titanium homogeneous structure, copings, the laser power should not be too large, affecting the scanning speed of the organizational structure is weak, relatively speaking, the thickness has a certain influence on the organizational structure.

\section{References}

[1]Contreras EF, Henriques GE, Giolo SR, Nobilo MA. Fit of cast commercially pure titanium and Ti-6Al-4V alloy crowns before and after marginal refinement by electrical discharge machining[J].J Prosthet Dent, 2002, 88(5): 467-472

[2]Zein I, Hutmacher DW, Tan KC, Teoh SH. Fused deposition modeling of novel scaffold architectures for tissue engineering applications. Biomaterials, 2002, 23(4):1169-85

[3]Santos EC, Osakada K, Shiomi M. Microstructure and mechanical properties of pure titanium models fabricated by selective laser melting[J]. Journal of Mechanical

Engineering Science, Proceedings of ImechE,2004,218C(7):711-719

[4]Peng L, Taiping Y, Sheng L. Direct laser fabrication of nickel alloy samples[J]. Int J Mach Tools Manuf,2005,45(11):1288-94

[5]Hollander DA, Walter MV, Wirtz TT. Structural, mechanical and in vitro characterization of individually structured Ti-6Al-4V produced by direct laser forming[J].Biomaterials,2006, 27:955-963 